\title{
Study on UAV Flight State Simulation
}

\author{
Sun Yong, Li Wen-wei, Song Yi, Meng Hong, Li Zeng-lu \\ China Research and Development Academy of Machinery Equipment
}

\begin{abstract}
Flight state is an important research content of UAV technology, it involves many factors, and simulation is very difficult. Based on the knowledge of relevant mathematical knowledge and flight control theory, a UAV flight control simulation method is proposed. The method combining sampling step and Gaussian white noise sampling values, UAV flight state parameters at each sampling point and output of the flight state parameters are obtained by iterative methods. This method is simple and practical, and it meets the needs of the UAV flight state simulation in the large-scale system simulation.
\end{abstract}

Keywords: UAV, Flight State, Simulation

\section{Preface}

UAV simulation plays an important role in the system simulation. The UAV flight state simulation is an important part of the UAV simulation. In large-scale system simulation, on the one hand, we should ensure that the simulation system and the actual system is basically similar in function, and guarantee the credibility of the simulation results, on the other hand, we should ensure that the simulation system is as simple and reliable as possible, and ensure that the simulation system efficient running on the computer. Consider the existing UAV simulation study focused on the theoretical aspects, including UAV control algorithms, navigation algorithms, intelligent optimiza- tion algorithms, and other theoretical studies. The currently UAV flight state theory and simulation theory is mostly too complex and difficult to achieve. These can not meet the needs of largescale system simulation.

In order to solve the above problems, a UAV flight state simulation method is proposed based on relevant mathematical knowledge and flight control theory, the method calculates the UAV flight information of the state and output information, including the position, speed and attitude angle. A practical and reliable UAV state simulation model is established and it meets the application requirements.

\section{Simulation method}

The simulation process of the UAV reconnaissance flight state at any time:

Get the initial pitch and roll angle of UAV, and UAV initial heading angle is obtained according to the UAV preset speed;

The UAV planning route point is sampling in every preset sampling step. Combined with the sampling step, Gaussian white noise sampling value and UAV preset speed, UAV pitch and roll angle are obtained at each sampling point with the iterative manner;

According to the sampling step, Gaussian white noise sampling values, and UAV preset speed, the UAV heading angle and simulation total speed $r$;

After UAV pitch, roll and heading angle are determined, combined with the UAV initial position, the UAV position 
information and the speed are obtained at each sampling point with iterative manner.

The each steps of the simulation method are described in detail as follow.

\section{Simulation of UAV flight state}

The following instructions for UAV flight state parameters in any sampling time. Initially, set any point position in UAV reconnaissance routes is $\left(\mathrm{X} 1_{t}, Y 1_{t}\right.$, $\mathrm{Z1}_{\mathrm{t}}$ ), and set the UAV speed in the location is $\left(\mathrm{V}_{\mathrm{X} 1 \mathrm{t}}, \mathrm{V}_{\mathrm{Y} 1 \mathrm{t}}, \mathrm{V}_{\mathrm{Z} 1 \mathrm{t}}\right)$, $\mathrm{T}$ is the sampling step, $\mathrm{W}_{\mathrm{i}}$ is white Gaussian noise sampling value with mean 0 and variance 1 .

Considering UAV performance and environmental factors, UAV fly in accordance with the planned trajectory is impossible. The UAV autopilot control pitch and roll, and navigation system control the heading.

Remains in a stable flight state, autopilot stable UAV flight in the same horizontal position (pitch angle and roll angle are 0 ), set stable error mean is 0 , standard deviation are $\sigma_{\varepsilon}, \sigma_{\gamma}$, correlation coefficients are $\rho_{1 \mathrm{~T}}, \rho_{2 \mathrm{~T}}$, at any time the aircraft's pitch angle and roll angle can be calculated:

$$
\begin{aligned}
& \Delta \varepsilon_{0}=0 \\
& \varepsilon_{t}=0+\Delta \varepsilon_{t} \\
& \Delta \varepsilon_{t}=e^{-\rho 1_{T} \bullet T} \cdot \Delta \varepsilon_{t-1}+\sqrt{1-e^{2 \rho 1_{T} \bullet T}} \cdot \sigma_{\varepsilon} \cdot W_{i} \\
& \varepsilon_{t}=e^{-\rho 1_{T} \bullet T} \cdot \Delta \varepsilon_{t-1}+\sqrt{1-e^{2 \rho 1_{T} \bullet T}} \cdot \sigma_{\varepsilon} \cdot W_{i} \\
& \Delta \gamma_{0}=0 \\
& \gamma_{t}=0+\Delta \gamma_{t} \\
& \Delta \gamma_{t}=e^{-\rho 2_{T} \bullet T} \cdot \Delta \gamma_{t-1}+\sqrt{1-e^{2 \rho 2_{T} \bullet T}} \cdot \sigma_{\gamma} \cdot W_{i} \\
& \gamma_{t}=e^{-\rho 2_{T} \bullet T} \cdot \Delta \gamma_{t-1}+\sqrt{1-e^{2 \rho 2_{T} \bullet T}} \cdot \sigma_{\gamma} \cdot W_{i}
\end{aligned}
$$

When UAV navigation system can error-free control UAV in accordance with the preset heading, UAV faceted navigation error mean is 0 , standard deviation is $\sigma_{\beta}$, the correlation coefficient is $\rho_{3 \mathrm{~T}}$, at any reconnaissance moment, the UAV's heading angle is:

$$
\begin{aligned}
& \beta_{t}=\frac{V_{X 1_{t}}}{\sqrt{V_{X 1 t}^{2}+V_{Y 1 t}^{2}}}+\Delta \beta_{t} \\
& \Delta \beta_{t}=e^{-\rho_{3 T} \cdot T} \cdot \Delta \beta_{t-1}+\sqrt{1-e^{2 \rho_{3 T} \cdot T}} \cdot \sigma_{\beta} \cdot W_{i}
\end{aligned}
$$

Set $\Delta \beta_{0}=0$

When UAV speed remains the same, the speed control error mean is 0 , mean square deviation is $\sigma_{V}$, correlation coefficient is $\rho_{4 \mathrm{~T}}$, at any time of the UAV reconnaissance aircraft, the simulation total speed is:

$V_{t}=\sqrt{V_{X 1 t}^{2}+V_{Y 1 t}^{2}+V_{Z 1 t}^{2}}+\Delta V_{t}$

$\Delta V_{t}=e^{-\rho_{4_{T}} \cdot T} \cdot \Delta V_{t-1}+\sqrt{1-e^{2 \rho_{4_{T}} \cdot T}} \cdot \sigma_{V} \cdot W_{i}$

Set $\Delta \mathrm{V}_{0}=0$

As UAV in actual flight speed may be changes in real time, therefore its position may deviate from the default route of flight. Based on UAV previous sampling position, UAV heading angle and speed in this sample, UAV position in this sample can be acquired by the following formula.

$$
\begin{aligned}
& X_{t}=X_{t-T}+V_{t} \cdot \cos \beta_{t} \cdot \cos \varepsilon_{t} \cdot T \\
& Y_{t}=Y_{t-T}+V_{t} \cdot \sin \beta_{t} \cdot \cos \varepsilon_{t} \cdot T \\
& Z_{t}=Z_{t-T}+V_{t} \cdot \sin \varepsilon_{t} \cdot T \\
& X_{0}=X_{1_{0}}, Y_{0}=Y_{1_{0}}, Z_{0}=Z_{1_{0}}
\end{aligned}
$$

Which, $\left(X_{1_{0}}, Y_{1_{0}}, Z_{1_{0}}\right)$ is the coordinates in the UAV take-off point. UAV position information that obtained in this way is closer to the actual situation. UAV speed on the level of $\mathrm{X}, \mathrm{Y}$ direction and perpendicular to the $\mathrm{Z}$ direction, respectively:

$V_{X_{t}}=V_{t} \cdot \cos \beta_{t} \cdot \cos \varepsilon_{t}$

$V_{Y_{t}}=V_{t} \cdot \sin \beta_{t} \cdot \cos \varepsilon_{t}$

$V_{Z_{t}}=V_{t} \cdot \sin \varepsilon_{t}$ 
UAV flight position $\left(X_{t}, Y_{t}, Z_{t}\right)$ and flight speed $\left(V_{X_{t}}, V_{Y_{t}}, V_{Z_{t}}\right)$ obtained by the above formula is the UAV flight simulation state results.

\section{Simulation of the UAV flight state output}

In reconnaissance process, the UAV can send the current flight output parameter state to the ground by data link (e.g., sent to the ground intelligence processing vehicle), for the positioning of the reconnaissance target. Due to the impact of the UAV performance and environmental factors, flight state parameters of UAV output and UAV's actual flight state parameters will have large errors. Following UAV flight state output parameter is simulated combined with circular error probability and high measurement error when UAV position, UAV output location information, pitch angle, roll angle and heading angle are get. Flight state parameters UAV output include three positions and three attitude angles, UAV three positional parameters correspond to the three UAV coordinates values in threedimensional coordinate system, while the UAV three attitude angles are pitch angle, roll angle and heading angle.

Set UAV positioning error circular error probability is $\sigma_{\mathrm{D}}$ (meters), correlation coefficient is $\rho_{5 \mathrm{~T}}$, the height measurement error variance is $\sigma_{\mathrm{H}}(\mathrm{m})$, correlation coefficient is $\rho_{6 \mathrm{~T}}$. According to the abovedescribed manner, the UAV flight condition parameter at any sampling time can be obtained. UAV reconnaissance positioning information is $\left(X_{t}, Y_{t}, Z_{t}\right)$, the speed is $\left(V_{x t}, V_{Y t}, V_{Z t}\right)$ at any sampling time, set:

$$
\begin{aligned}
& \Delta D_{t}=e^{-\rho_{s \supset} \cdot T} \cdot \Delta D_{t-1}+\sqrt{1-e^{2 \rho_{s t} \cdot T}} \cdot \sigma_{D} \cdot W_{i} \\
& X_{t}^{\prime}=X_{t}+\Delta D_{t} \cdot \frac{V X_{t}}{\sqrt{V X_{t}^{2}+V Y_{t}^{2}}}
\end{aligned}
$$

$$
\begin{aligned}
& Y_{t}^{\prime}=Y_{t}+\Delta D_{t} \cdot \frac{V Y_{t}}{\sqrt{V X_{t}^{2}+V Y_{t}^{2}}} \\
& \Delta Z_{t}=e^{-\rho_{6 \tau} \bullet T} \cdot \Delta Z_{t-1}+\sqrt{1-e^{2 \rho_{67} \bullet T}} \cdot \sigma_{Z} \cdot W_{i} \\
& Z_{t}^{\prime}=Z_{t}+\Delta Z_{t}
\end{aligned}
$$$$
\left(X_{t}^{\prime}, Y_{t}^{\prime}, Z_{t}^{\prime}\right) \text { is UAV position- }
$$

ing information, which is UAV output itself position in the three-dimensional coordinate system.

Assumes that the UAV autopilot stabilize the aircraft in the horizontal position (pitch and roll angle is 0), the stability error mean is 0 , variance are $\sigma_{\varepsilon}$ and $\sigma_{\gamma}$ respectively, correlation coefficient are $\rho_{7 \mathrm{~T}}$ and $\rho_{8 \mathrm{~T}}$ respectively. The output pitch angle and roll angle of the UAV at any reconnaissance time:

$$
\begin{aligned}
& \varepsilon_{t}=e^{-\rho_{7 \tau} \bullet T} \cdot \Delta \varepsilon_{t-1}+\sqrt{1-e^{2 \rho_{77} \bullet T}} \cdot \sigma_{\varepsilon} \cdot W_{i} \\
& \gamma_{t}=e^{-\rho_{87} \bullet T} \cdot \Delta \gamma_{t-1}+\sqrt{1-e^{2 \rho_{8 T} \bullet T}} \cdot \sigma_{\gamma} \cdot W_{i}
\end{aligned}
$$

Assumed that the UAV navigation error mean is 0 and variance is $\sigma_{\beta}$, correlation coefficient is $\rho_{9 \mathrm{~T}}$, the output heading angle of UAV at any reconnaissance time: $\beta_{t}^{\prime}=\beta_{t}+\Delta \beta_{t}$ $\beta_{t}=\frac{V X_{t}}{\sqrt{V X_{t}^{2}+V Y_{t}^{2}}}$ $\Delta \beta_{t}=e^{-\rho_{9 T} \bullet T} \cdot \Delta \beta_{t-1}+\sqrt{1-e^{2 \rho_{9 T} \bullet T}} \cdot \sigma_{\beta} \cdot W_{i}$ $\left(\beta_{t}^{\prime}, \varepsilon_{t}, \gamma_{t}\right)$ are three attitude angles of UAV output.

The UAV output of the positioning information $\left(X_{t}^{\prime} 、 Y_{t}^{\prime} 、 Z_{t}^{\prime}\right)$ and three attitude angles $\left(\beta_{t}^{\prime}, \varepsilon_{t}, \gamma_{t}\right)$ are obtained by the above formula. That is the simulation of the UAV output flight state parameters. As considering the circular probability error and height measurement error of UAV devices in the positioning, UAV output state parameters, which are obtained in this way, is similar with the UAV output flight state parameters of actual situation. 


\section{Conclusion}

Based on the relevant mathematical knowledge and flight control theory, an UAV flight state simulation method is proposed, the method is combined with sampling step, Gaussian white noise sampling values. Flight state parameters and output of the flight state parameters of UAV at each sampling point can be obtained by iterative methods. Through experiments proved that the method is simple, practical, and able to meet the needs of large-scale system simulation.

\section{References}

[1] ZHENG Tao, GAO Zheng-hong, KONG Xiang-jun. Research on Simulation and Modeling for Unmanned
Aerial Vehicle System. Flight Dynamics. 2002, 20(3).

[2] WANG Jin-yun, WEI Rui-xuan, DONG Zhi-xing, etc. Research on Formation Flight Control of Cooperative UAV. Fire Control \& Command Control. 2010, 35(3).

[3] JIANG Hao-liang. UAV flight control system simulation. Nanjing University of Science and Technology. 2004

[4] TAO De-gui, LIU Zhi-qiang, QIAN Wei. Design and Implementation of UAV Flight Training Simulation System. Computer Simulation. 2008, 25(3).

[5] SHUI Wei, GE Yan, HAN Yu, etc. Path Planning for UAV Based on Mixed Ant Colony Algorithm. Journal of System Simulation. 2011, 23(3). 